\section{On repositioning customer support services: some food for further thought}

Repositioning customer support services

1809

The framework proposed by Sheth et al. (2020) (hereafter SJA) for repositioning customer support services (CSS) is a timely and thought-provoking contribution to the extant literature. The need for a change in the corporate mindset and direction vis-à-vis CSS that SJA advocate (succinctly captured in their Figures 1 and 2), as well as their seven recommendations for engineering such a change (Figure 3) are logical and insightful. In the concluding section of their paper SJA call for additional research and allude to three broad areas worth investigating. This brief commentary builds on and augments SJA's research agenda by highlighting three additional critical areas stemming from their proposed framework and recommendations.

First and foremost, I believe it is important to distinguish clearly between "customer [support] service" [singular] and "customer support services" [plural], a distinction that at times gets blurred when the terms are used interchangeably. A strong focus on delivering consistently superior customer service, which is a corporate cultural trait often difficult to cultivate, can be a powerful and profitable source of competitive advantage to any company, regardless of company type (e.g. B2B vs B2C), size, offerings, support services, etc. (Berry, 1999). However, the ROI for efforts to develop and nurture a company-wide, unwavering commitment to excellent customer service may be more difficult to demonstrate compellingly (at least in the short term) than is the ROI for developing specific customer support services. This potential challenge raises several interrelated research-worthy questions in the context of "repositioning CSS from an administrative cost center to a strategic profit center" as advocated by SJA. For instance:

RQ1. What is the nature of the association between a company's generic customer service level and the effectiveness (and eventual profitability) of its specific CSS?

RQ2. Given the presumed difficulty in assessing ROI for generic service excellence relative to assessing ROI for specific CSS, are companies likely to overemphasize the latter at the expense of the former?

RQ3. Is there a minimum threshold level of generic service that needs to be in place before investments in specific CSS start to pay off?

RQ4. Can increasing the number and variety of CSS offered compensate for shortfalls in generic customer service?

Second, the provision of customer service as well as the delivery of products and services (including CSS) are increasingly being handled by service ecosystems, each consisting of multiple distinct organizations that are interlinked is some fashion to cater to the same focal customers. The growing prevalence of service ecosystems in catering to customers introduces a new layer of complexity that offers additional

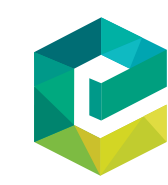

European Journal of Marketing Vol. 54 No. 7,2020 pp. $1809-1811$ (c) Emerald Publishing Limited DOI 10.1108/EJM-07-2020-973 
avenues for scholarly inquiry vis-à-vis SJA's recommendations for repositioning CSS. For instance:

- Which entity (or entities) within the ecosystem should take the lead in championing the proposed corporate shift towards viewing CSS through a strategic-profit lens rather than an administrative-cost lens?

- What are the challenges and opportunities vis-à-vis coordinating the generation and dissemination of customer insights across entities within the ecosystem?

- Are there feasible and effective approaches for melding corporate cultures, frontline employee training, IT support systems, etc., across entities within the ecosystem so as to create a seamless service experience for customers?

Third, the notion of understanding and managing customer experience throughout the entire customer journey - from pre-purchase to during purchase/consumption to postpurchase - is garnering growing attention in both business and scholarly literatures. For instance, in a comprehensive conceptual piece on this topic, Lemon and Verhoef (2016) propose and discuss in detail a "process model for customer journey and experience." This model delineates various customer touchpoints before, during and after purchase (within a given episode/experience as well as across episodes/ experiences over time). One important aspect of this model that is intertwined with the preceding discussion of SJA's proposal for repositioning CSS in the context of service ecosystems is that Lemon and Verhoef:

- categorize customer touchpoints at each of the three purchase stages (pre-, during and post-) into brand-owned, partner-owned, customer-owned or external/social touchpoints, implying potential shifts in the locus of control and responsibility for the customer's experience during their journey; and

- offer a rich agenda for further research in this domain.

The challenges posed by possible changes in ownership of the customer's experience in the course of their journey are akin to the ecosystem-related issues raised previously. As such, yet another potentially fruitful avenue for scholarly inquiry is to re-examine SJA's seven recommendations for repositioning CSS by juxtaposing them against Lemon and Verhoef's research agenda, as well as service ecosystem-related issues such as the ones raised earlier. A systematic comparative re-examination of SJA's recommendations along those lines is likely to generate new insights about contextual factors and contingencies on which the degree of success in the implementing each of the recommendations might depend.

In conclusion, SJA's proposed framework and recommendations for repositioning CSS constitute a valuable addition to the current literature on customer service as noted at the outset of this commentary. What SJA advocate however also raises several key additional issues that warrant further scholarly inquiry to generate a richer, more nuanced set of insights for effectively transitioning from the conventional administrative-cost-center view of CSS to the proposed strategic-profit-center view. 


\section{References}

Berry, L.L. (1999), Discovering the Soul of Service: The Nine Drivers of Sustainable Business Success, The Free Press, New York, NY.

Lemon, K.N. and Verhoef, P.C. (2016), "Understanding customer experience throughout the customer journey”, Journal of Marketing, Vol. 80 No. 6, pp. 69-96.

Repositioning

customer

support

services

Sheth, J.N., Jain, V. and Ambika, A. (2020), "Repositioning the customer support services: the next frontier of competitive advantage", European Journal of Marketing, forthcoming.

1811 